\title{
Otros dibujos, misma realidad. \\ Del proyecto de Felipe León Maffey a la copia de Sebastián Feringán Cortés para el acueducto de Cartagena
}

Other drawings, same reality. From the Felipe León Maffey project to the copy of Sebastián Feringán Cortés for the Cartagena aqueduct

V ALeria Manfrè Universidad de Valladolid 


\section{RESUMEN}

En este artículo se analiza el proyecto realizado en 1724 por el ingeniero italiano Felipe León Maffey para la reparación de la cañería del acueducto de la ciudad de Cartagena a partir de la copia realizada, en 1732, por el ingeniero Sebastián Feringán Cortés (17001762). El proyecto, además, se completa con cuatro dibujos, copias de Feringán Cortés, que se han estudiado en relación con las fuentes textuales.

Palabras clave

Cartagena, Acueducto, Dibujo, Felipe León Maffey, Sebastián Feringán Cortés, Ingenieros militares.

Abstract

This article focuses on the project carried out in 1724 by the Italian engineer Felipe León Maffey, to repair the plumbing of the aqueduct of the city of Cartagena, from the copy made, in 1732, by the engineer Sebastián Feringán Cortés (1700-1762). The project is also completed with four drawings, copies of Feringán Cortés, which have been studied in relation to textual sources.

KEY WoRDS

Cartagena, Aqueduct, Drawing, Felipe León Maffey, Sebastián Feringán Cortés, Military Engineers.

Recibido: 16 de enero de 2020. Aceptado: 12 de marzo de 2020.

El presente estudio se ha realizado gracias a la financiación del Ministerio de Ciencia, Investigación y Universidades (Programa Juan de la Cierva-Incorporación) en el marco del Proyecto de Investigación I+D del Ministerio de Ciencia, Investigación y Universidades (HAR2016-79442-P), Hacia Antonio Acisclo Palomino. Teoría e historiografía artísticas del Siglo de Oro. 
A principio del siglo XvI, el crecimiento demográfico de Cartagena y la designación como puerto militar de Castilla en el Mediterráneo había favorecido la realización de obras hidráulicas para solventar el problema del escaso abastecimiento de agua potable. Debido a este inconveniente, el capitán general de la mar, don García de Toledo, había dejado de invernar las galeras en el puerto de Cartagena. En 1564, ante esta dificultad, el rey Felipe II incitó al Concejo a emprender una serie de obras para aumentar la disponibilidad hídrica y encauzar las fuentes en torno a los parajes de San Juan, El Calvario y el Barranco del Feo'.

Los trabajos parecen empezar durante los primeros meses de 1577 y la búsqueda de nuevas fuentes no resultó suficiente hasta que, en 1587, se optó por traer agua desde los ríos granadinos Castril y Guardal, una labor interrumpida y empezada de nuevo en 1617.

Finalmente, debido a la complejidad de las obras, muy ambiciosas y quizá por su elevado coste, en 1640 las autoridades decidieron interrumpir las labores $^{2}$. Durante el siglo XVII los proyectos de trasvase se verán afectados por diferentes motivos (guerras y dificultades económicas) y la conducción de agua no se había llevado a efecto. Será solamente con la nueva política de fomento patrocinada por los Borbones cuando se volvió a retomar el tema; de hecho, entre 1742 y 1743, el ingeniero Sebastián Feringán Cortés (1700-1762)³ — personaje

1 Alejando Egea Vivancos, Laura Arias Ferrer, «El abastecimiento de agua a la ciudad de Cartagena en época moderna: los manantiales de San Juan, La Cruz, el Calvario y el Barranco del Feo a la luz de los textos y la arqueología», Revista Murciana de Antropología, 14 (2007), págs. 259-282; págs. 272-273.

2 Egea Vivancos, Arias Ferrer, «El abastecimiento; págs. 279-280.

3 Sobre Sebastián Feringán Cortés remitimos a Pedro A. Berenguer Ballester, Documentos y noticias para la biografía del General de Ingenieros D. Sebastián Feringán y Cortés, Madrid, Imp. del Memorial de Ingenieros, 1896; Antonio Bethencourt, «El Escorial y la construcción del arsenal de Cartagena por D. Sebastián Feringon», Boletín del Seminario de Arte y Arqueología, XXVIII (1962), págs. 298-302; José Ma. DE Jaime LoRén, «Sebastián Feringán y Cortés (Báguena, 1700-1762). Arquitecto e ingeniero militar, constructor del Arsenal de Cartagena entre otras importantísimas obras», Xiloca, 15 (1995), págs. 207-231, donde se hace referencia a los planos de Simancas; Horacio CAPEL, «Los proyectos del ingeniero militar Sebastián Feringan (1741) y de Francisco Boizot (1774) para el trasvase de los ríos Castril y Guardal con la construcción de un canal navegable y para el riego de los campos de Lorca, Totana, Cartagena y Murcia», Biblio 3W. Revista Bibliográfica de Geografía y ciencias sociales, 310, (2001), s.p; Álvaro Piñeras, Piñeras Rivas, «El ingeniero militar Sebastián Feringán, constructor del Real Arsenal de Cartagena», Revista de Historia Naval, III, 8 (1985), págs. 111-139 y María Jesús Peñalver Martínez, Juan Francisco Maciá Sánchez, «Los proyectos de 
del que nos ocuparemos más adelante- entregaría unos planos, perfiles y una memoria donde apuntaba a la importancia de tal proyecto. Sin embargo, pasaron los años y al llegar el siglo Xx se prescindió de la idea del trasvase hacia la ciudad de Cartagena ${ }^{4}$.

Volviendo al siglo XVII, sabemos que durante los años se suceden algunos proyectos para remodelar el puerto, aunque escasos parecen ser los estudios historiográficos sobre el abastimiento de agua a través del manantial cerca de Cartagena, una cuestión que, con toda seguridad, tuvo que seguir siendo de gran interés y debate. Aun así, no se había llegado a ningún tipo de solución durante la época de Carlos II cuando, en 1668, Cartagena se convirtió en base permanente de la Escuadra de Galeras ${ }^{5}$. De esta época proliferan los proyectos para el fondo de la bahía y de sus dimensiones, incluyendo sondeos y medidas para ampliar el puerto, como lo demuestra el plano de la dársena realizado por el ingeniero militar Lorenzo Possi a instancia del Marqués del Viso, capitán general de la Escuadra de Galeras de España, y que finalmente fue desestimado por el Consejo de Guerra, no tanto por su deficiencia técnica, sino por su alto coste ${ }^{6}$. A esta propuesta siguió el plano de Juan Bautista Balfagón de 1677 que, como el anterior, contenía intervenciones para adecuar las instalaciones portuarias de Cartagena, como los sondeos y medidas del puerto, que tampoco se llegaría a materializar ${ }^{7}$.

Durante el gobierno de los Borbones se impulsaron una serie de reformas de mayor envergadura para modernizar la Marina y colocarla al mismo nivel que las potencias europeas. En 1726, bajo el impulso de José Patiño, Intendente General de la Marina Española de Felipe V, se crearon los Departamentos Marítimos, dividiendo el litoral español en Norte, Mediodía y Levante con sus respectivas capitales en El Ferrol, Cádiz y Cartagena ${ }^{8}$. El objetivo del gobierno

\footnotetext{
los ingenieros militares Alejandro de Rez y Antonio Montaigú de la Perille para el puerto de Cartagena. Las primeras propuestas para la transformación de su contorno litoral (1717-1731)», Scripta Nova, vol. XVII, 437 (2013), s.p. Por último remitimos a Horacio CaPEL, et al., Los Ingenieros militares en España. Siglo XVIII. Repertorio biográfico e inventario de su labor científica y espacial, Barcelona, Universidad de Barcelona, 1983, págs. 170-178.

4 El proyecto de Feringán no se realizó, y la puesta en práctica del proyecto tuvo que esperar hasta los años setenta del siglo XVIII. Sobre la evolución de este trasvase remitimos a CAPEL, «Los proyectos, s.p.

5 José María Rubio Paredes, Álvaro de la Piñera Rivas, Los ingenieros militares en la construcción de la Base Naval de Cartagena (siglo XVIII), Madrid, Servicio de Publicaciones del EME, 1988, pág. 63.

6 Juan Francisco Maciá Sánchez, María J. Peñalver Martínez, Francisco Segado Vázquez, «Los diseños realizados para la construcción del Real Arsenal de Cartagena (1670-1731). Un paradigma del conocimiento ilustrado", en Santiago Huerta (ed.), Actas del Séptimo Congreso Nacional de Historia de la Construcción, Madrid, Instituto Juan de Herrera, vol. 2, 2011, págs. 809-820; pág. 811.

7 Peñalver Martínez, Maciá Sánchez, «Los proyectos», s.p.

8 Rubio Paredes, de la Piñera Rivas, Los ingenieros, pág. 59-61.
} 
borbónico, tras el nombramiento en 1728 de Cartagena como capital departamental de Levante, fue convertir la ciudad en una base naval fortificada. Esta decisión implicó para Cartagena la necesidad de concebir una serie de estudios para la construcción de nuevas instalaciones e infraestructuras necesarias para la Armada Real; in primis, la construcción de la dársena artificial que serviría para ampliar la capacidad del puerto natural de la ciudad y de un arsenal ${ }^{9}$. Este suceso favoreció, además, la llegada a la ciudad de diferentes ingenieros que se dedicaron a levantar varios planos de la bahía. De la amplia documentación gráfica sobre las instalaciones portuarias y de las intervenciones para la construcción del arsenal ha hablado extensamente la historiografía española ${ }^{10}$. Sin duda alguna, fue uno de los mayores retos al que se enfrentaron las autoridades a lo largo del siglo XVIII. Para la construcción portuaria la ubicación elegida fue la zona denominada como Mar de Mandarache, al noreste del puerto, y fueron involucrados algunos de los mejores ingenieros y técnicos de la época, como Alejandro de Rez y Antonio Montaigú de la Perille, cuyos primeros proyectos, presentados en 1728, atestiguan la intensa actividad centrada en la instalación militar para la construcción y el mantenimiento de buques.

La actividad cartográfica de época borbónica de los ingenieros militares sigue estando relacionada también con el problema del aprovisionamiento de agua que se tradujo en una representación gráfica de los espacios territoriales de la mano de dos ingenieros, el casi desconocido Felipe León Maffey (Mafey, Mafei) ${ }^{11}$, y el español Sebastián Feringán Cortés. Ambos se dedicaron a las tareas de levantamiento cartográfico de amplios territorios de Cartagena desarrollando una cartografía temática, la hidráulica. Sobre este material aquí esbozado y casi inexplorado, dedicaremos las siguientes líneas.

\section{Felipe León Maffey: aproximación bibliográfica}

La campaña de renovación, saneamiento, y propuestas relativas a la ciudad, el puerto y sus infraestructuras de Cartagena había empezado en 1720 cuando, por voluntad de Felipe V, Cartagena fue nombrada Base Naval para la Escuadra de Galeras de España. El año siguiente, el ingeniero general de la

\footnotetext{
9 María J. Peñalver Martínez, La génesis de la dársena del puerto de Cartagena a lo largo del siglo XVIII, Tesis doctoral, Universitat Politècnica de Cartagena, 2 vols., 2012.

10 Peñalver Martínez, Maciá Sánchez, «Los proyectos», s.p.

11 Hemos optado por esta grafía, en lugar de Mafey o Mafei, porque es la que hemos encontrado en los dibujos firmados por el ingeniero.
} 


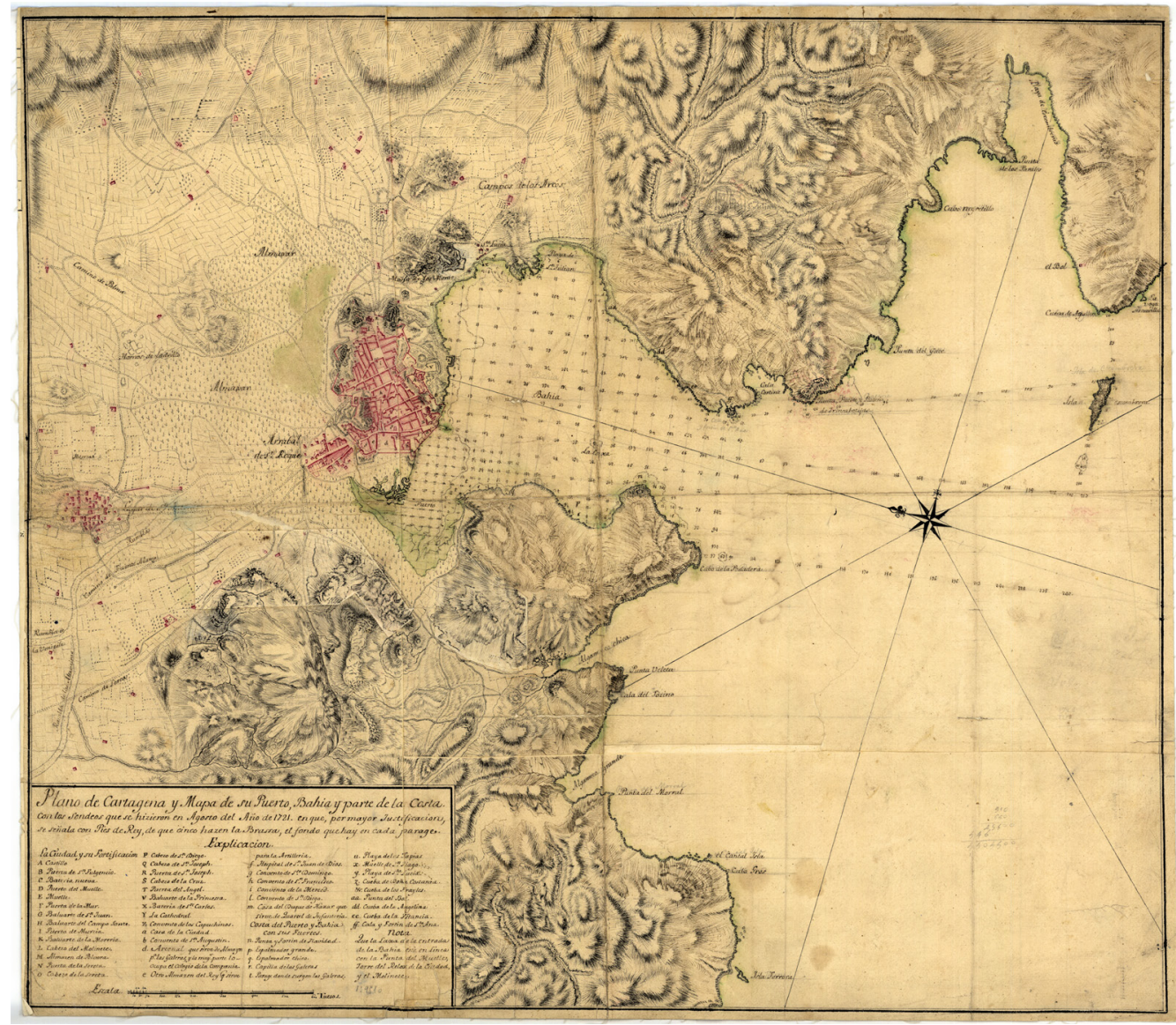

Fig. 1. Anónimo, atribuido a Felipe León Maffey, Plano de Cartagena y mapa de su Puerto y parte de la Costa con los sondeos que se hizieron en Agosto del año 1721, en que, por mayor justificación, se señala con Pies de Rey, de que cinco hacen la brassa, el fondo que hay en cada parage, 1721, España. Ministerio de Defensa. Instituto de Historia y Cultura Militar. Archivo Cartográfico y de Estudios Geográficos del Centro Geográfico del Ejército de Madrid, Ar.G-T.4.C.1-43

corona española Jorge Próspero Verboom ${ }^{12}$, acompañado por su hijo y también ingeniero, Isidro Próspero, se trasladaron a Cartagena con el fin de elaborar

12 Sobre la figura de Verboom, creador del cuerpo de ingenieros del ejército a comienzos del siglo xviII, remitimos a Juan Miguel Muñoz Corbalán, Jorge Próspero Verboom, ingeniero militar flamenco de la monarquía hispánica, Madrid, Fundación Juanelo Turriano, 2015. Sobre la figura de los ingenieros en el siglo de las Luces véase Antoine Picon, Architectes et ingénieurs au siècle des Lumières, Marseille, Éditions Parenthèses, 1988. 
un plan para la configuración del territorio. Con la llegada de los ingenieros Verboom se empiezan a realizar nuevos planos de la bahía y, durante el mes de agosto de 1721, bajo la probable dirección de Isidro, el ingeniero Felipe León Maffey entregaba un Plano de Cartagena, y Mapa de su Puerto, Bahia y parte de la Costa $[\ldots]^{13}$. Aunque las pruebas no son explícitas ni precisas, esta supuesta paternidad del plano de Maffey apunta hacia la hipótesis de que ya, en 1721, se encontraba en Cartagena, implicado en la restructuración de la bahía (Fig. 1).

Previamente, antes de su llegada a Cartagena, el ingeniero Maffey, de origen italiano ${ }^{14}$, estaba trabajando en la construcción de la ciudadela de Barcelona, momento en el que fue designado — tal y como indica la minuta de un memorial fechado 21 de julio de 1717 y firmado por Verboom, Patiño y el comandante Jean François de Bette, III marqués de Lede ${ }^{15}$ — para incorporarse a la expedición de Italia, durante la guerra de la Cuádruple Alianza (1717-1720), urdida por el monarca Felipe $\mathrm{V}$ con el objetivo de reconquistar las islas de Cerdeña y Sicilia. De esta expedición hemos localizado un plano manuscrito de la ciudad de Cagliari firmado por Maffey, que ofrece una síntesis interesante del territorio de la ciudad al que hemos dedicado un estudio reciente ${ }^{16}$.

Sabemos que después de la conquista de la isla en 1717 por parte del ejército de Felipe V, Maffey se quedó en Cerdeña para realizar obras de fortificación, pero nada más podemos añadir sobre su trayectoria profesional en la península italiana ${ }^{17}$. Tras desarrollar su labor en Cerdeña, experiencia que le sirvió para adiestrar sus habilidades y actitudes, y una supuesta estancia en Sicilia, volvemos a tener noticias suyas a partir del año 1721, durante su estancia en Cartagena, involucrado en las obras del acueducto de la ciudad.

Estos años previos a su llegada a la costa cartaginense fueron decisivos para el aprendizaje en materia científica y técnica del ingeniero italiano. El ambiente fue muy favorable para una inmersión en el mundo de la guerra y para el desarrollo de su personalidad y facultades técnicas y manuales a través de las acciones bélicas sobre el terreno, una preparación previa de la que se pudo

13 José María Rubio Paredes, Cartagena. Puerto de Mar, Madrid, Lunwerg Editores y Autoridad Portuaria de Cartagena, 2005, pág. 184; Peñalver Martínez, Maciá Sánchez, «Los proyectos, s.p. El plano anónimo se encuentra en el Centro Geográfico del Ejército de Madrid, a la signatura Ar.G-T.4.C.1-43. Existe otro plano anónimo, de la misma fecha, y casi idéntico a la signatura Ar.G-T.4.C.1-44.

14 Capel, et al., Los Ingenieros, pág. 293.

15 Archivo General Militar de Madrid (en adelante AGMM), Campaña de Italia, siglo XVIII, 7391.2.

16 Valeria Manfrè, «Una isla y sus imágenes. La cartografía militar de Cerdeña (1717-1720)», en Mercedes Gómez Ferrer, Yolanda Gil Saura (eds.), Ecos culturales, artísticos y arquitectónicos entre Valencia y el Mediterráneo en Época Moderna, Valencia, Universitat de Valencia, 2018, págs. 207-225, pág. 213.

17 Durante la investigación llevada a cabo en archivos y bibliotecas de Cagliari no se ha localizado ningún mapa o documento que se relacione con el ingeniero Maffey. 
aprovechar en los años siguientes y que se manifiesta en la notable destreza que contienen los dibujos para el proyecto del acueducto de Cartagena.

\section{Del proyecto de Maffey a las copias de Feringán Cortés}

En los años siguientes a la designación de Cartagena como Base naval en 1720 , se realizaron nuevos informes, planos y elevaciones relacionados con la labor de mantenimiento de las conducciones de las aguas, algunas subterráneas y abovedadas, y otras dirigidas a la reparación de las cañerías, que llevó a la plasmación de nuevos dibujos e informes de época borbónica. Esta labor fue llevada a cabo por el ingeniero Maffey que en Cartagena continuó completando su larga carrera profesional de la que, al día de hoy, desconocemos su formación teórica. Durante su estancia en la ciudad, Maffey fue involucrado en las obras de remodelación del acueducto para la canalización de las aguas del manantial de los Dolores ${ }^{18}$, y otras instalaciones militares, como la Casa del Rey (Fig. 2) ${ }^{19}$.

Un análisis comparativo entre los documentos relativos al proyecto y al plan de trabajo con especificaciones técnicas y los dibujos, permite apreciar y ubicar el papel, tanto de Maffey, como de la participación del ingeniero Feringán Cortés.

La actividad de Maffey como ingeniero en Cartagena se remonta, como hemos aludido antes, al año 1721, periodo del que, supuestamente, nos ha llegado el primer testimonio gráfico: un mapa de la ciudad. El cometido inicial de Maffey fue el de efectuar un minucioso reconocimiento y estudio del terreno que se dispondría a realizar para las autoridades. Con este fin llevaría a cabo un proyecto para el acueducto de Cartagena, encargado por el teniente general conde de Rivere, al que se adjuntaba un peritaje general del mismo, ambos fechados el 8 de marzo de 1724. Por último, dedicó su atención a redactar un plan de trabajo que acabaría durante el mes de mayo del mismo año. Estos documentos iban acompañados por unos planos, alzados y secciones del terreno, donde se representaron las nuevas obras del acueducto por realizar, así como donde ejecutar nuevas labores para el restablecimiento de la conducción del agua y sus respectivas arcas.

18 El nombre del manantial, desde la documentación manejada, no queda reflejado. Sin embargo, parece que las aguas de Los Dolores se utilizaban para la navegación y para las galeras atracadas en el puerto. Egea Vivancos, Arias Ferrer, «El abastecimiento, pág. 278 y Vicente Montojo Montojo, «Obras camineras, portuarias y de abastecimiento de agua en el siglo XVIII », Historia de Cartagena, 8 (2000), págs. 85-92, pág. 90.

19 Las remodelaciones del complejo de la Casa del Rey, centro de actividad militar y utilizado, entre otras cosas, para el servicio de artillería o como fábrica de pólvora, será objeto de futuras investigaciones. 

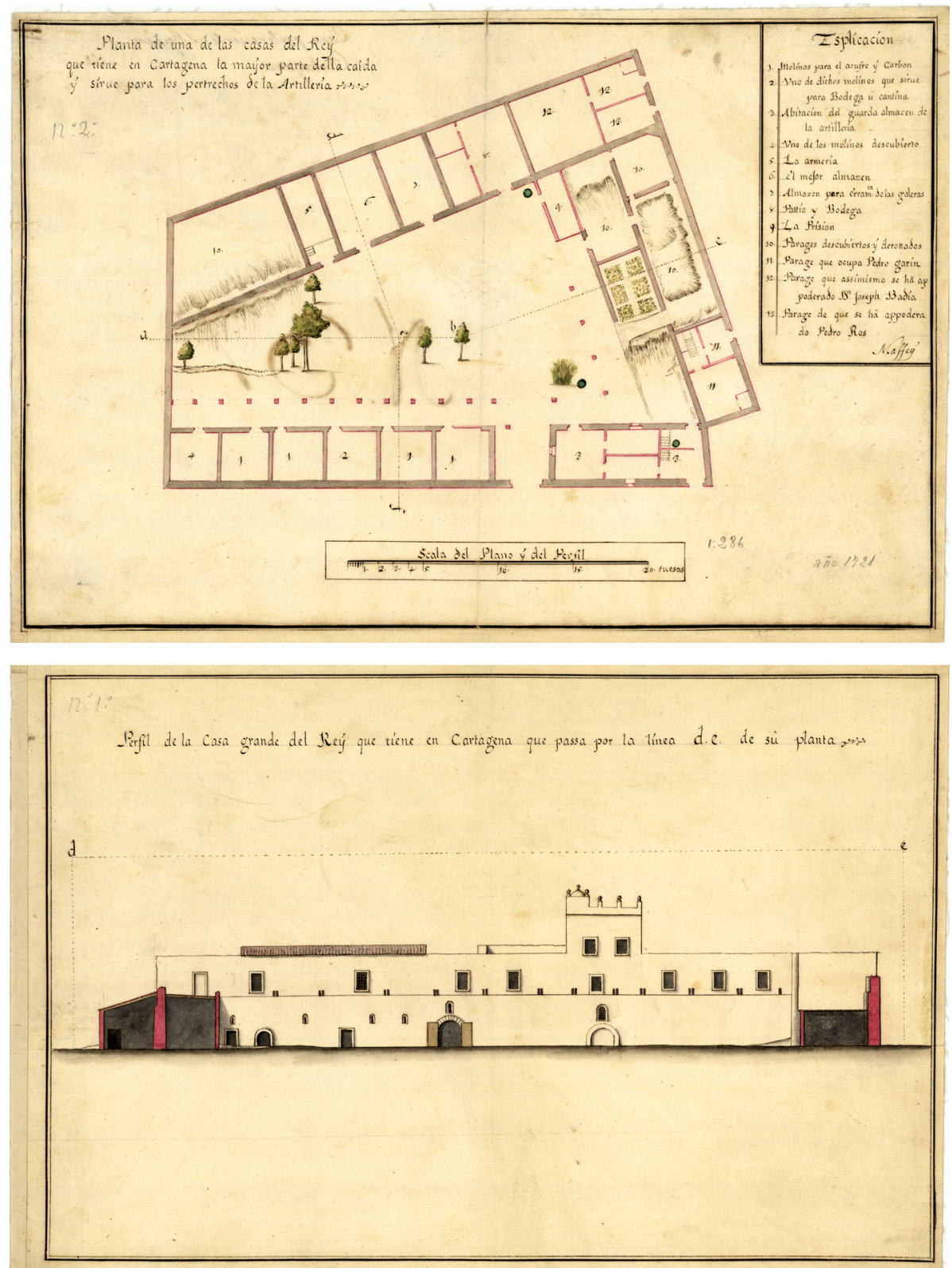

$\uparrow \rightarrow$ Fig. 2. Felipe León Maffey (atribuido), Planta de una de las casas del Rey que tiene en Cartagena la mayor parte della caída... 1721, España. Ministerio de Defensa. Instituto de Historia y Cultura Militar. Archivo Cartográfico y de Estudios Geográficos del Centro Geográfico del Ejército de Madrid, Ar.G-T.4.C.1-47 
Jirgil de la casa grande del Keý que tiene en Carragena, que passa por la linea abs de si jlanta
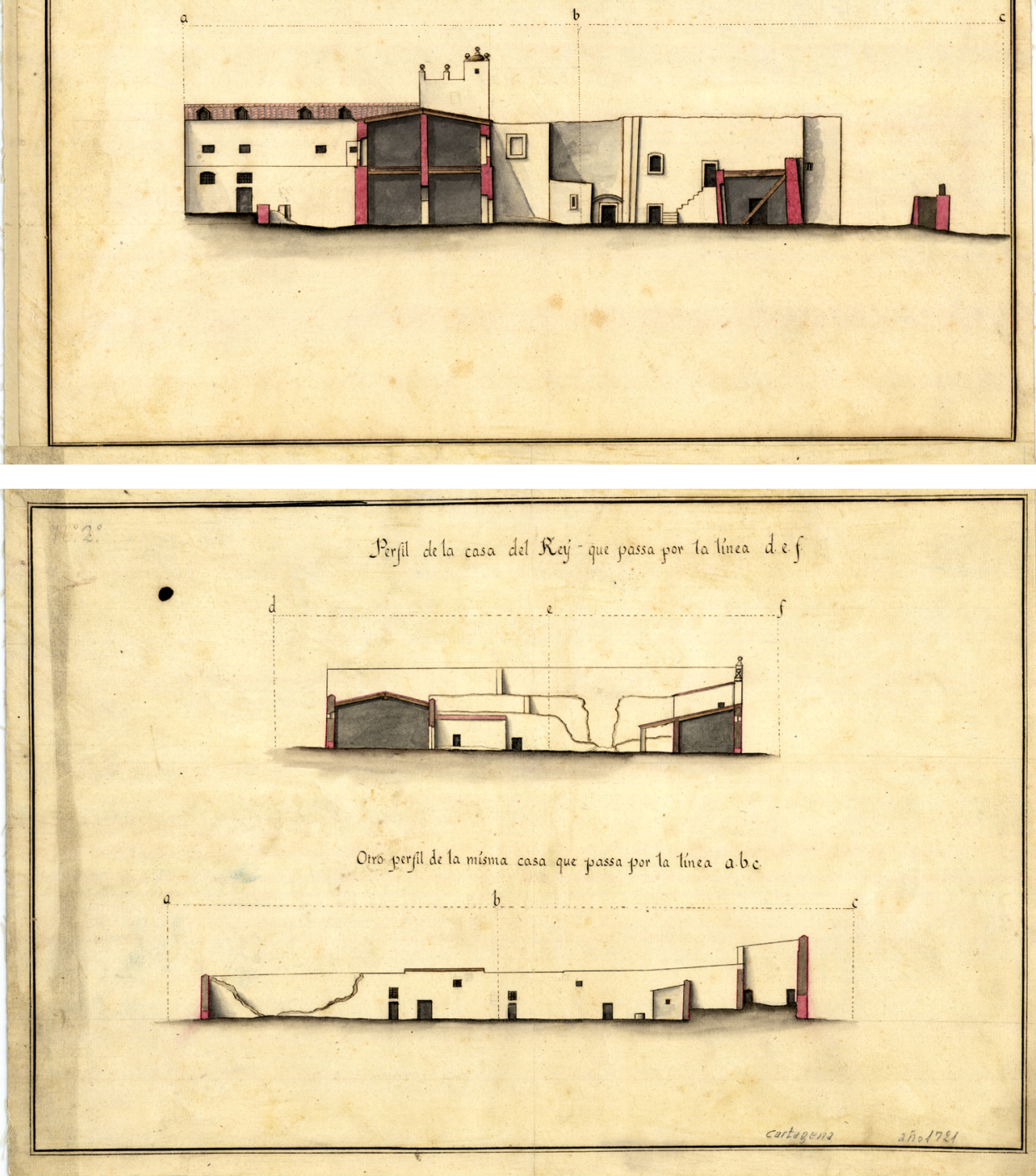

Fig. 2. [Continuación] 
El contenido de los tres documentos, que resume el pragmatismo y el espíritu racional típico de los ingenieros, muestra el solvente trabajo de recopilación de datos de carácter técnico por parte de Maffey, que elaboró en su propuesta un plan de reformas que giraba, básicamente, en torno a la reparación de la cañería, su mantenimiento y, en su caso, la construcción de diferentes porciones del acueducto, con sus respectivas arcas. El proyecto se titula Valutazion y Condizion para la construcción de la cañería que conduze el agua a las fuentes de esta Ciudad de Cartagena, cuias estimaciones, disposiciones, y proyecto con los Planos, Perfiles y elebaziones que ban adjuntos a esta, son copia de los que para el mas seguro azierto de tan importante obra formó el Ingeniero Don Phelipe de Leon y Mafey ${ }^{20}$.

De los tres documentos redactados por Maffey para la planificación de este valioso recurso hidráulico, hemos localizado las copias fechadas el 29 de diciembre de 1732 y realizadas por el ingeniero Sebastián Feringán Cortés, que enviaría el 31 de diciembre del mismo año desde Cartagena a don José Patiño.

Previamente hay que aclarar que la copia del tercer documento termina con una anotación de Feringán en el que se hacía referencia a los cuatro planos, perfiles y elevaciones realizados por Maffey y que se adjuntaban en copia a la documentación, con el fin de ser analizados. De hecho, como veremos, el mismo Maffey en su larga disertación, hace referencia, en más de una ocasión, a sus dibujos.

Los testimonios gráficos de mano del ingeniero italiano sobre este proyecto y evaluación inicial de la cañería del acueducto de Cartagena, que demostrarían su destreza gráfica a la hora de realizar los perfiles del terreno y la elevación de las partes que componen el acueducto, parece que se hayan extraviado o, por lo menos, hasta la fecha no se han localizado. De hecho, el mismo Feringán advertía que, tanto el proyecto con su estimación, como los cuatro dibujos, «son copia de los papeles originales, que he hecho en virtud de orden del excelentisimo señor Don Joseph Patiño» ${ }^{21}$, cumpliendo con los dictamines emanados de su superior y comunicándoselos a través del gobernador de la plaza de Cartagena, don Juan Antonio de Ornedal y Maza.

Así que, para documentar la obra del acueducto, se realizaría una segunda narración gráfica, tras la realizada y acabada por Maffey en 1724, efectuada por Feringán que, al enviar la copia del proyecto de Maffey de 1732 a Patiño, con el fin de ser evaluados, adjuntó la documentación gráfica que hemos localizado, junto con el proyecto, en el Archivo General de Simancas.

20 Archivo General de Simancas (en adelante AGS), Secretaría de Marina (en delante SMA) leg. 0375, s.f.

${ }^{21}$ AGS, SMA, leg. 0375, s.f. 


\section{El proyecto}

A continuación realizaremos un análisis comparativo entre el contenido del proyecto de Maffey y los planos, perfiles y elevaciones que lo acompañan. En primer lugar, el ingeniero italiano empezaba su disertación haciendo referencia a la fecha de encargo por parte de las autoridades para estudiar el estado del acueducto. A partir del 12 de noviembre de 1723, Maffey centró sus esfuerzos en llevar a cabo una exploración topográfica para el conocimiento del territorio, indispensable para el abastecimiento de agua, así como para averiguar la calidad y la cantidad de agua del que disponía Cartagena. Las reflexiones de carácter práctico se basaron en un reconocimiento in situ tanto del manantial, como del acueducto, al que se añadía un exhaustivo estudio de medición del terreno. El acueducto resultaba en algunas partes quebrado y arruinado, de este modo en el proyecto se proponía reedificar la cañería de este tramo para asegurar el abastecimiento de agua potable a toda la ciudad y a las embarcaciones que invernaban en el puerto ${ }^{22}$.

Refiriéndose a estos reparos el protagonismo de Maffey se expresó en la planificación general de la obra y dejó realizado un plano de la plaza de Cartagena y del terreno «en que se comprehende el surfidero, o manantial y el camino que haze el aquiducto, hasta comunicar las aguas a todas las nueve fuentes de esta Ziudad ${ }^{23}$. Las primeras consideraciones del detallado estudio indicaban que una porción del acueducto se encontraba en buen estado y el ingeniero italiano alegaba que para obviar a la obstrucción del conducto era necesaria una simple limpieza de la cañería «en la qual puede haverse intriduzido algún ympedimento al curso de las aguas, que es desde el Manantial hasta l'Arca, que en el Plano está señalada con la letra D [...]». Respetando el orden alfabético, texto y plano quedaban relacionados por las correspondientes llamadas que aclaraban el estudio realizado por Maffey.

En la descripción de estos elementos y para aclarar el contenido del proyecto es de gran ayuda el Plano de Cartagena en el qual se describe el camino del Aqueducto $i$ sus Fuentes ${ }^{24}$, que debería corresponder al que hace referencia Maffey en su informe (Fig. 3).

$22 \quad$ AGS, SMA, leg. 0375, s.f.

23 AGS, SMA, leg. 0375, s.f.

24 AGS, Mapas, Planos y Dibujos (en adelante MPD), 16, 042, 26,2 x 40,5 cm. El plano ha sido publicado en Antonio José Mula Gómez, Juan Hernández Franco, «Los abastecimientos de agua potable en la Región de Murcia: una visión histórica», en Antonio Gil Olcina (ed.), La Cultura del Agua en la Cuenca del Segura, Murcia, Fundación CajaMurcia, 2004, págs. 167-198: págs. 178 y 179. 


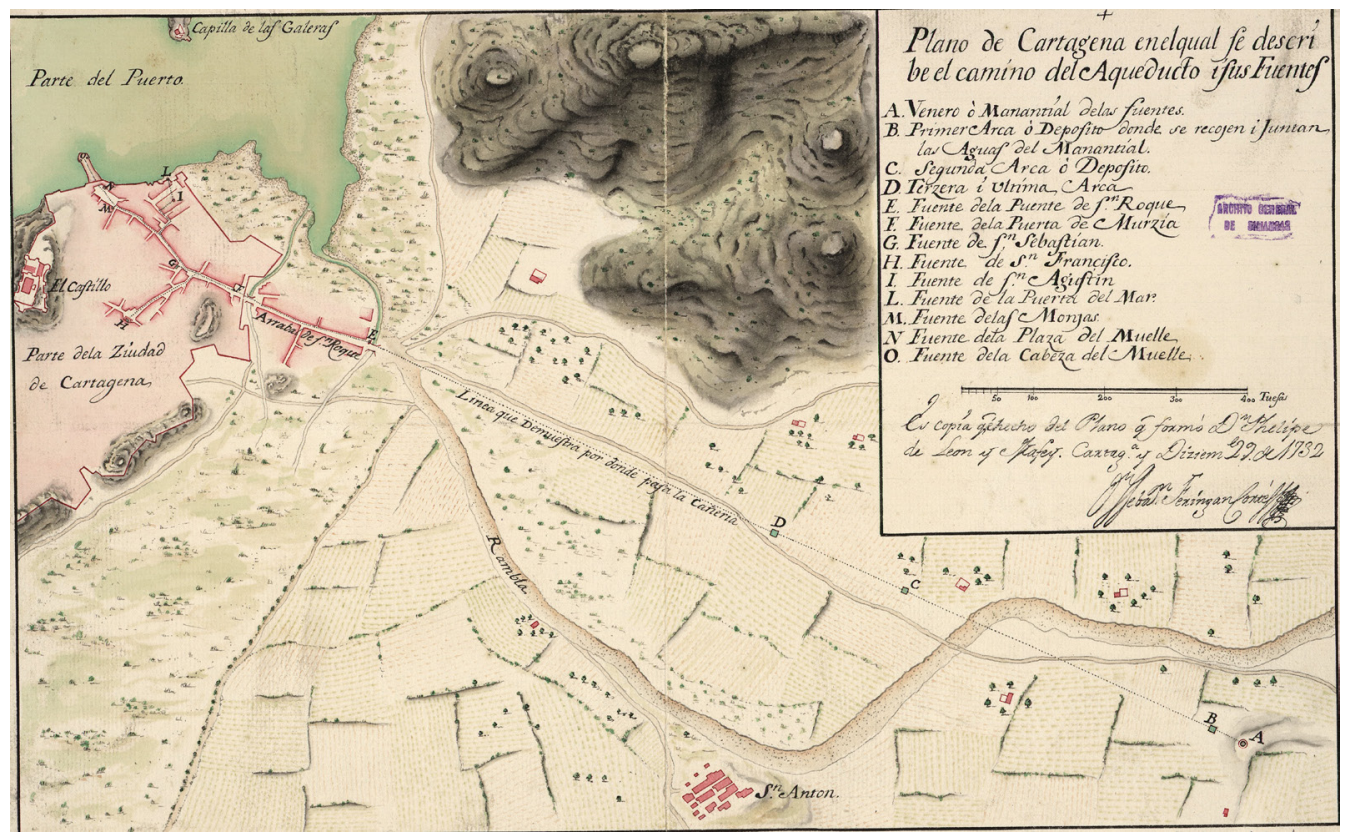

Fig. 3. Felipe León Maffey, copias de Sebastián Feringán Cortés, Plano de Cartagena en el qual se describe el camino del Aqueducto i sus Fuentes, 1732, España, Ministerio de Educación,

Cultura y Deporte. Archivo General de Simancas, Mapas, Planos y Dibujos, 16, 042

Como se ha señalado anteriormente, este plano es una copia realizada por Feringán, de hecho, en la leyenda situada en la parte derecha del plano, por debajo de donde se expresa la escala del mismo (400 toesas), hay una rúbrica de Feringán que recita: «es copia qe hecho del Plano q formó D.n Phelipe de Leon y Mafey. Cartag.a y Diziem.e 29 de 1732». En el plano manuscrito en aguada de gran belleza con colores verde, azul, gris, ocre y encarnado se representa la vegetación, los cultivos y los relieves por sombreado para crear sensación de volumen. En la leyenda, con clave alfabética la letra «A» corresponde al venero de las fuentes, las letras «B», «C $\mathrm{C}$ « «D» corresponden a las respectivas arcas o depósitos donde se recogen las aguas del manantial. El informe, además, aclaraba que este tramo del acueducto se encontraba construido bajo tierra y, desde el manantial hasta la última arca (la «D»), «se necesita construir un Arca principal o conservatorio, a quarenta y cinco o cinquenta tuesas del dicho manantial», con el fin de recoger todas las aguas. Además, era indispensable construir otras tres arcas inferiores con piedra de sillería que se realizarían al mismo nivel y cerca de las otras arcas ya existentes que estaban, en parte, arruinadas y de calibre insuficiente para que el agua pudiese fluir con mayor velocidad. 


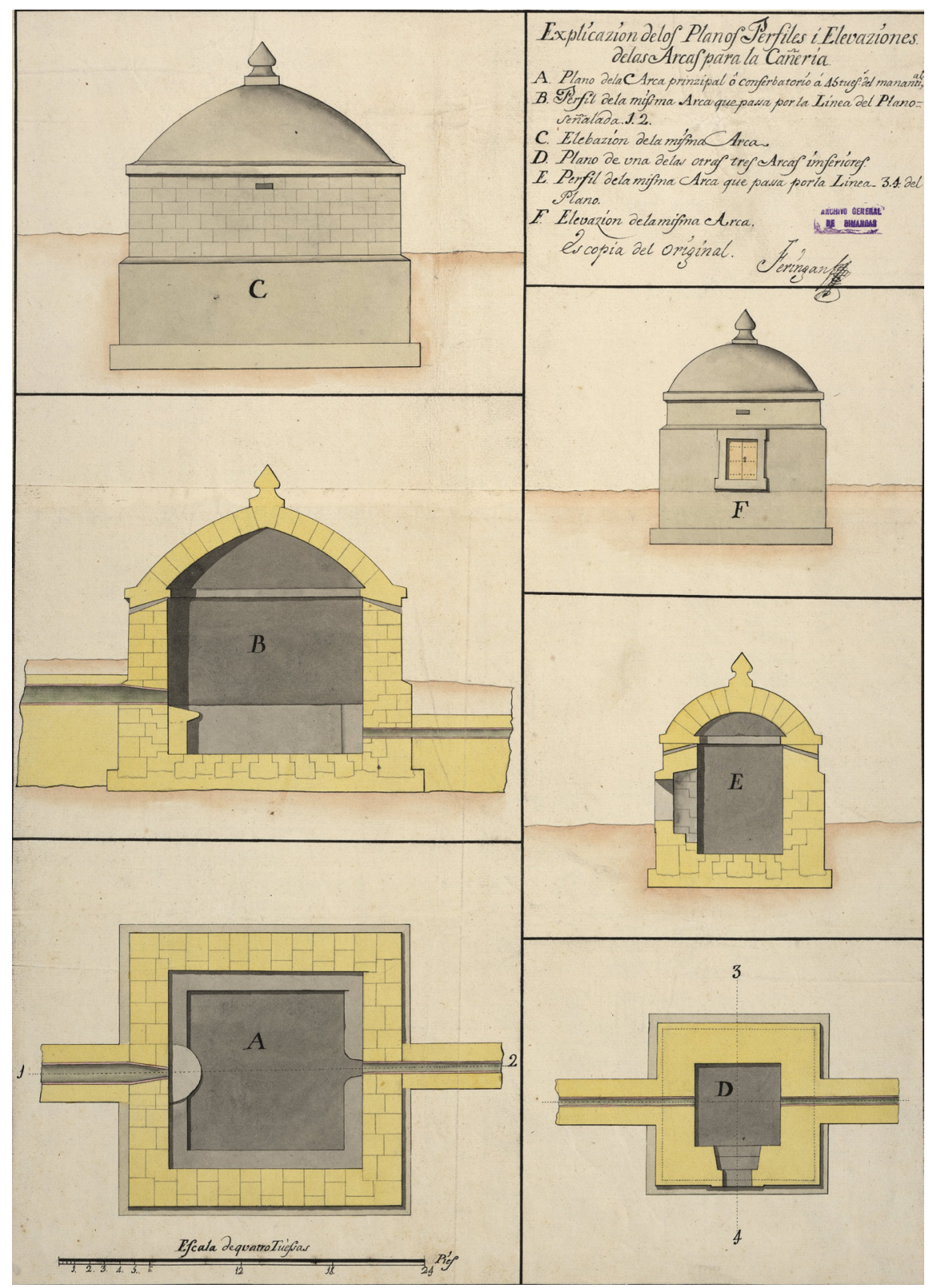

Fig. 4. Felipe León Maffey, copias de Sebastián Feringán Cortés, Explicación de los planos, perfiles y elevaciones de las arcas para la cañería de la ciudad de Cartagena, 1732, España, Ministerio de Educación, Cultura y Deporte. Archivo General de Simancas, Mapas, Planos y Dibujos, 25, 004 
Para la construcción del arca principal que debía servir como depósito de agua, y las restantes tres arcas inferiores, Maffey aconsejaba de utilizar sillares de buena calidad para poder soportar cualquier tipo de eflorescencias de salitre y al informe añadía una contribución dibujada, la Explicación de los planos, perfiles y elevaciones de las arcas para la cañería de la ciudad de Cartagena (Fig. $4)^{25}$, del que poseemos una copia hecha por Feringán. El dibujo tiene la finalidad principal de mostrar los aspectos fundamentales para abordar en las obras y representa la planta, sección y alzado, tanto del arca principal (a la izquierda), como de una de las otras tres arcas.

Además, los datos reunidos por Maffey indicaban que a partir de la última arca, la «D», hasta el puente del arrabal de San Roque, barrio construido desde 1541 al Norte de la rambla de Santa Florencina (futuro barrio del Carmen) ${ }^{26}$, tanto el acueducto que pasaba bajo tierra, como la cañería, estaban totalmente arruinados, causando derrame de agua, inutilizable, además, por su salubridad; lo mismo pasa con el último tramo, es decir, desde el arrabal hasta llegar al muelle de Cartagena, donde la cañería en muchas partes también estaba dañada y esto causaba que durante todo el año en las fuentes de este tramo escasease agua. El completo plan de reforma, según Maffey, para que todas las nueve fuentes de la ciudad tuviesen agua potable para el alivio del pueblo y decoro de la ciudad se reduciría a

[...] construir de nuevo todo el adquiduto desde la ultima arca $\mathrm{D}$, hasta la Puente del Arraval de San Roque así los cimientos como la muralla que deve sostener, la cañería de buena mazoneria, dandole suficiente espesor y profundidad de tal forma que la cañería, y porcion del adquidutto queden fuera del terreno a medida de lo que permitiere la desigualdad del terreno segun se expresa en su elevazion de los adjuntos divujos, con lo qual se consigue mayor solidez en dicho adquiduto y permanencia de su cañeria lo que no se podría conseguir, llevandola por devajo de tierra menos de fundar y fabricarle dentro del agua ${ }^{27}$.

Los nuevos cimientos del acueducto construido bajo tierra perderían resistencia y su estructura seguiría estando afectada por el peso del transito tanto de personas como de carruajes, aparte del peso del agua durante los días de lluvia intensa, así que los diferentes reparos argumentados en el informe deberían acotar estos problemas. Para obviar estos inconvenientes era necesario cons-

25 AGS, MPD, 25, 004, $51 \times 38 \mathrm{~cm}$.

26 Egea Vivancos, Arias Ferrer, «El abastecimiento, pág. 260.

27 AGS, SMA, leg. 0375, s.f. 
truir el acueducto, en las áreas que lo permitiesen, fuera del terreno y la cañería de nueva construcción debía ser de mejor calidad respecto al material que se había empleado anteriormente. En lo referente al primer aspecto, la solución propuesta contemplaba también evitar gastos excesivos o innecesarios durante eventuales obras de mantenimiento, como la revisión periódica del mismo o, en su caso, la reparación de las cañerías. De este modo, según el cálculo económico de Maffey, el coste sería mucho más asequible respecto a una construcción bajo tierra.

Pocos meses después de haber elaborado su proyecto, la mente racional de Maffey elabora otro documento de inestimable utilidad; el 10 de mayo de 1724, el ingeniero se encargaría de remitir a las instituciones de Cartagena un plan de trabajo riguroso y ordenado, dividido en veinticinco puntos, con el fin de explicar, paso por paso, en que manera y con qué orden deberían ser empezadas las obras del acueducto. Y, refiriéndose a este tramo concreto, es decir, a partir del arca «D» hasta el respiradero cerca del puente del arrabal, el documento empezaba apuntando que

Deve tener de profundo dicha escavazion hasta encontrar con el agua de incavo a otro, y de ancho, por la parte ynferior, lo que menos cinco piez, como se representa en el perfil $\mathrm{M}$ echando las tierras del lado que no embaraze el trasporte a los matteriales al tiempo de la construccion: siguiendo los alineamientos y reglas en dicha escavazion que por el sujeto o Persona a cuya direccion esté esta obra seran destinados ${ }^{28}$.

Para que fuese más comprensible Maffey creyó oportuno levantar y adjuntar un plano del tramo del acueducto, del que tenemos una copia realizada por Feringán, donde a la letra «M» de la leyenda correspondería cuanto indicado anteriormente en el texto, el «Perfil de las Excabaziones en que se demuestra lo ancho i profundo que proporcionalmente ba de tener» (Fig. 5) ${ }^{29}$.

Es fácil suponer que el propósito perseguido por Maffey, a través de este tercer documento, fue impedir interpretaciones variables en función de las diferentes actitudes de los ingenieros o, mejor dicho, de los técnicos responsables de la dirección de las obras sobre el terreno. Además, estas directrices permitirían ahorrar tiempo y gastos innecesarios gracias a la unificación de criterios de intervención, a pesar de las variables a las que podían enfrentarse los técnicos

28 AGS, SMA, leg. 0375, s.f. Divisas, y capitulaziones bajo las quales se deve componer y restablecer el aquiducto y cañeria que conduze las aguas a las nueve fuenttes de Carttaxena para que queden corrientes.

29 AGS, MPD, 16, 043, 29,7 x 40,5 cm. 


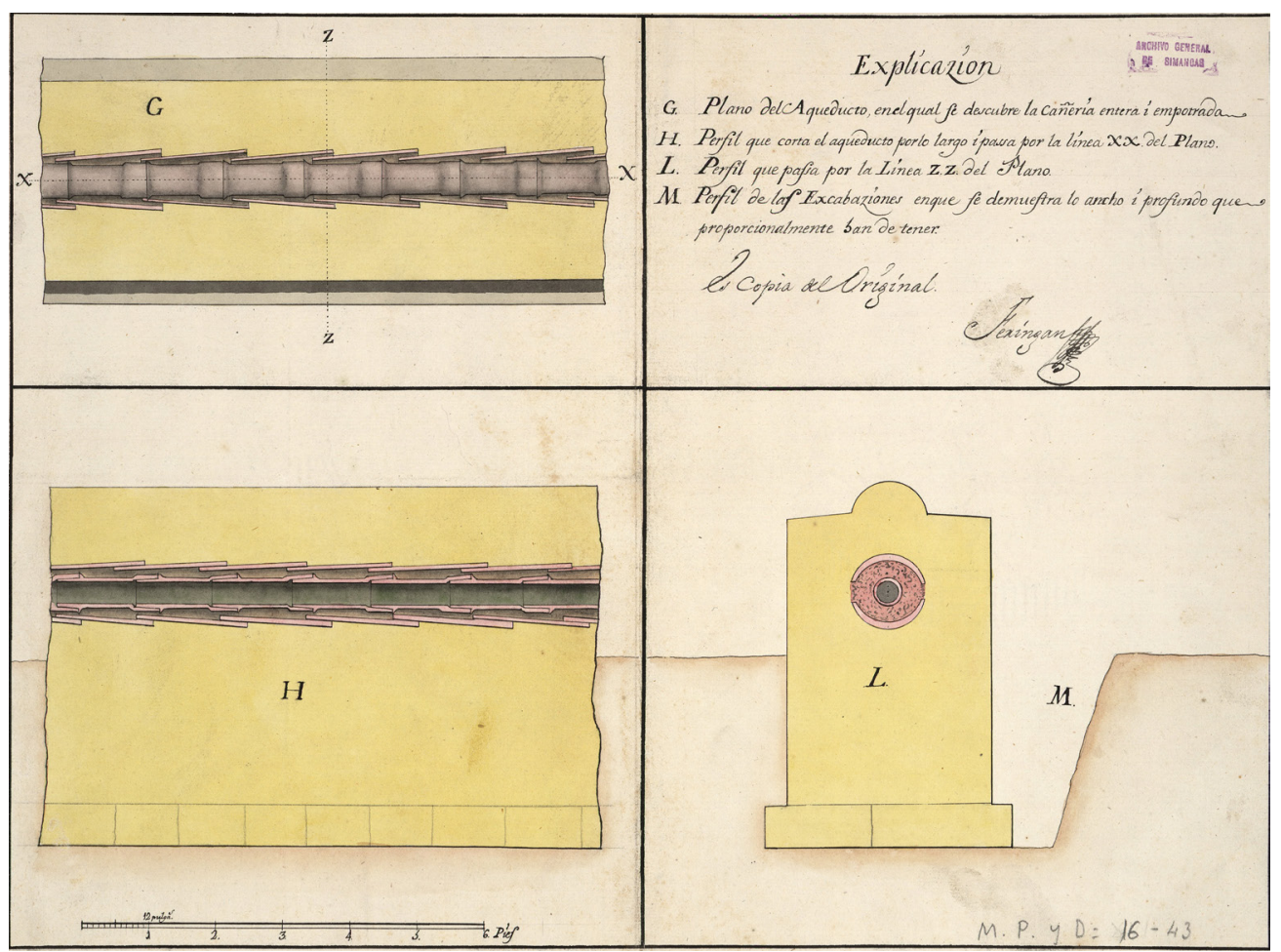

Fig. 5. Felipe León Maffey, copias de Sebastián Feringán Cortés, Explicación de los planos, perfiles y elevaciones de las arcas para la cañería de la ciudad de Cartagena, 1732, España, Ministerio de Educación, Cultura y Deporte. Archivo General de Simancas,

Mapas, Planos y Dibujos, 16, 043

durante la fase del trabajo. De este modo, las restantes materias tratadas, desde el punto dos en adelante, se refieren al momento siguiente, es decir a cuando se acabarían las excavaciones que supondrían la cimentación del acueducto; después se levantaría la muralla optando por el uso de mampostería, y allí se asentaría la cañería de tres pies de ancho con un declive adecuado para que las aguas no se detuviesen. Al concluirse el acueducto se seguiría con las excavaciones necesarias para conectarlo directamente con las nueve fuentes de la ciudad: las de San Roque, Puerta de Murcia, San Sebastián, San Francisco, Santa Catalina, Plaza del Rey, Puerta del Muelle, San Agustín y el Carmen ${ }^{30}$. Cuando la cañería construida hasta el puente del arrabal de San Roque estu-

30 Los documentos consultados no mencionan los nombres de las nueve fuentes que se han tomado desde el estudio de Montojo Montojo, «Obras camineras, pág. 90. 


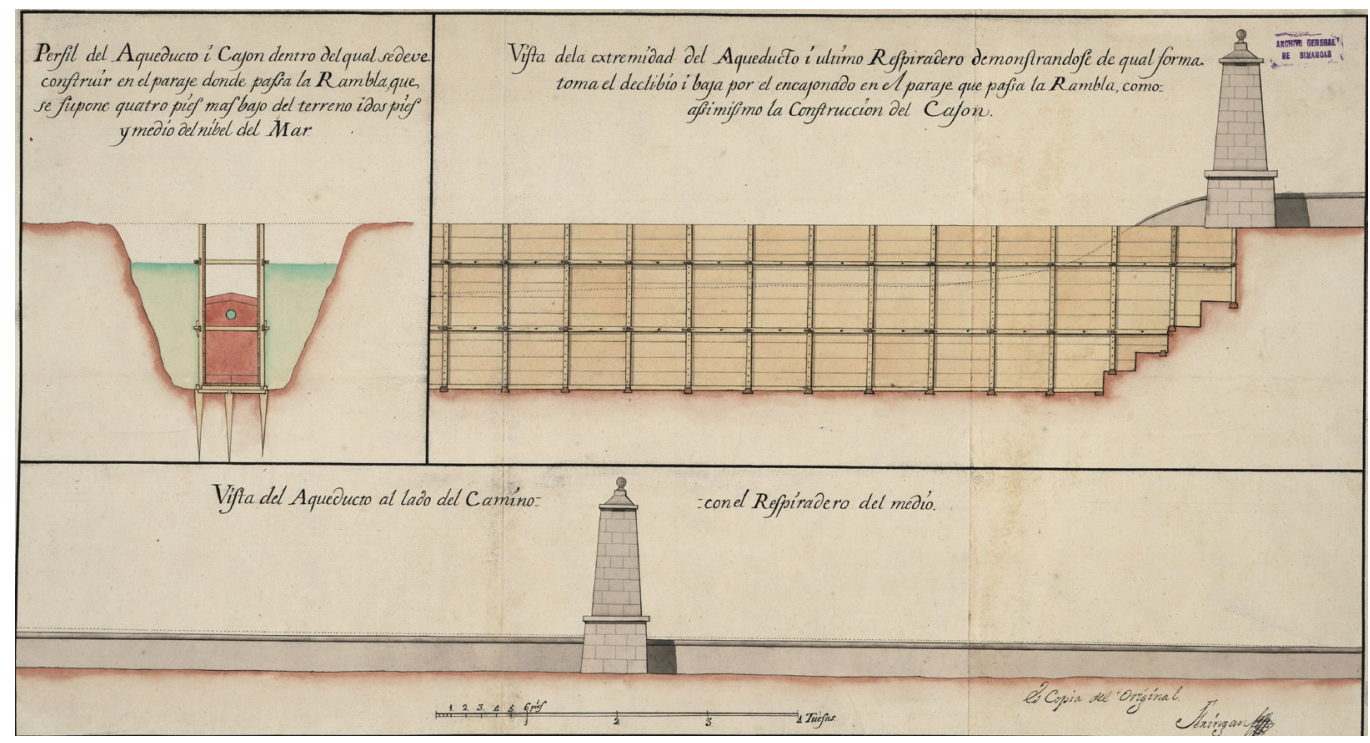

Fig. 6. Felipe León Maffey, copias de Sebastián Feringán Cortés, Perfil del acueducto y cajón dentro del cual se debe construir en el paraje donde pasa la rambla... y vistas del acueducto..., 1732, España, Ministerio de Educación, Cultura y Deporte. Archivo General de Simancas, Mapas, Planos y Dibujos 25, 003

viese acabada, se seguiría con las excavaciones del mismo acueducto desde el dicho arrabal hasta la puerta de Murcia, indicada en el plano de Cartagena con la letra «F». Además, respecto a este tramo del acueducto, Maffey señalaba que sería oportuno llevar a cabo una inspección para averiguar si la muralla podía sostener la nueva cañería que se debería construir ${ }^{31}$.

Asimismo, auspiciaba la construcción de cuatro respiraderos «de piedra de sillería, con un esperon a punta de Diamante», del que también nos ha llegado una copia realizada por Feringán (Fig. 6) ${ }^{32}$; tres de ellos se levantarían entre la arca «D»y antes de llegar al arrabal y el cuarto en la rambla que se encuentra a 354 toesas desde el manantial. La reparación, mejora e, incluso, transformación de algunas partes del acueducto debían estar a cargo de un empresario. En cuanto a los materiales que se utilizarían para la construcción de la nueva cañería, el ingeniero italiano aconsejaba traer todos los caños, o sea los conductos, desde la ciudad de Barcelona por ser de mejor calidad ${ }^{33}$.

31 AGS, SMA, leg. 0375, s.f.

32 AGS, MPD, 25, 003, $30 \times 50 \mathrm{~cm}$.

33 AGS, SMA, leg. 0375, s.f. A partir del punto 19 la documentación manuscrita indicaba la necesidad de utilizar cal, arena, y piedra de sillería de buena calidad en las obras del acueducto. 
Como hemos podido apreciar, Maffey, a lo largo del informe proporcionaba una serie de recomendaciones acerca de los materiales más aptos que habrían de utilizarse para la construcción. En la cultura técnica europea y en el ámbito de la ingeniería, o de la arquitectura, el conocimiento de los materiales que había de emplearse durante el proceso constructivo era un tema de primaria importancia y tiene una tradición extendida. En la tratadística clásica fue un tema señalado por Vitruvio en su tratado De Arquitectura (siglo I d.C.), y durante el Renacimiento no serían escasos los teóricos que, arrancando de Vitruvio, aludirían en sus textos a los materiales de construcción, como León Battista Alberti en su De re aedificatoria (1485), o en algunos apartados del Libro Primero del tratado I Quattro Libri dell'Architettura (1570) de Andrea Palladio.

En la parte final del proyecto, el ingeniero italiano aludía además a un documento de peritaje que él mismo realizo $\sigma^{34}$. Recurriendo a su experiencia, Maffey ejecutó una minuciosa planificación de los gastos y precisó las razones de esta estimación tan detallada; sospechaba de una cierta falta de confianza hacia estos técnicos que debían encargarse del peritaje de las obras y aludía a las dificultades para conseguir en la ciudad, o en sus inmediaciones, técnicos expertos en materia de estimación. Para evitar lo que había pasado en la anterior evaluación presentada por parte de unos albañiles que habían tasado toda la obra en 350 mil reales, pero proponiendo la mitad de los arreglos y obras propuestas por el italiano, éste pensó bien de realizar su propia estimación. Tras el análisis, la propuesta de Maffey supondría un gasto de 181938 reales y confiaba en que las obras estuviesen concluidas en ocho o diez meses ${ }^{35}$. Con este proyecto se completaba el objetivo de dotar finalmente de agua potable a la ciudad.

En síntesis, Maffey organizaba escrupulosamente las labores a desarrollar, y transmitía todo por escrito. A raíz de los documentos examinados, se observa la aplicada dedicación del italiano para el proyecto del acueducto, no realiza ningún tipo de evaluación superflua, y todo está planteado en términos objetivos. Asimismo, se demuestra muy minucioso a la hora de indicar el sistema constructivo, indicando las dimensiones de los nuevos canales o los materiales que habría que utilizar para el acueducto y su cañería.

Desde la lectura de los documentos algo más podemos extrapolar; deducimos que Maffey no se haría cargo de las labores, el sistema de puesta en obra debía ser llevado a cabo por sus colegas profesionales de la ingeniería militar, aún por determinar, y a quién se delegaría la responsabilidad de dirigir las obras

34 AGS, SMA, leg. 0375, s.f. Estivaciones y calculo del adquiducto con el detalle de lo que importara cada cosa en particular. Copia del original de Feringán firmada por Maffey del 8 de marzo de 1724.

35 En el proyecto Maffey da por concluidas las obras en diez meses mientras que, en el último documento redactado durante el mes de mayo, él mismo dice que las obras se deberán concluir en ocho meses. 
hidráulicas. Quizá esto justificaría la redacción de un proyecto tan detallado para ser posteriormente entregado a las autoridades de la ciudad.

Como ya hemos advertido, sobre Maffey son muy escasos los datos concretos sobre su trayectoria profesional y circunstancias personales, pero nuevos datos de archivo justifican sus indicaciones y consejos técnicos. En $1724^{36}$, con el grado de ingeniero en segundo, Maffey abandonaba Cartagena para dirigirse a Nueva España, un encargo al que probablemente estaba ya destinado en el momento de redactar el proyecto del acueducto, del que no se haría cargo. En la ciudad de Veracruz se ocupó de las fortificaciones de la ciudad, de las que nos ha llegado un mapa de 1726 y un proyecto para la reparación de la empalizada de la muralla de la ciudad que, en 1718, había sido afectada por el río Tenoya ${ }^{37}$.

Debido a la urgencia de las obras, y a juzgar por lo que sabemos hasta la fecha, es decir, a través de las cartas del regidor de la ciudad, don Pedro Frabega y Machabelo fechadas en $1732^{38}$, las obras para la reparación de la cañería del acueducto de Cartagena, se realizaron cuando era gobernador de Cartagena don Juan Antonio de Ornedal y Maza que, a su vez, había encargado la dirección de las obras a su secretario don Andrés Chereguini ${ }^{39}$, y a don Francisco Pinto ${ }^{40}$. Sin embargo, el regidor Frabega y Machabelo señalaba que estos dos individuos no habían estado a la altura de la realización de las obras de la cañería nueva por ser «sujetos nada inteligentes en obras», labores que «requieren continua aplicación y mucha practica ${ }^{41}$, reconociendo defectos, tanto en la cañería, como en las fuentes de la ciudad. De este modo aconsejaba la actuación inmediata de ingenieros con dilata experiencia para poder solventar, cuanto antes, el problema de la escasez de agua que seguía padeciendo el pueblo y la armada y que, por consiguiente, afectaba la actividad comercial. Resultaba necesario que las fuentes llevasen agua a todos los edificios del Arsenal, a la tripulación y a la guarnición de navíos y galeras. Esto explicaría el tenor de las copias de los despachos del Consejo de Castilla, fechados entre 1730 y $1732^{42}$, en donde se hacía referencia a los avisos del gobernador Ornedal y Maza, y precisaban la voluntad

36 AGMM, Colección Aparici, 1884, p. 107.

37 Ambos dibujos de Maffey se conservan en el Archivo General de la Nación en México y datan respectivamente, 1726 y 1727. Sobre estos dibujos véase David F. MARLEY, Historic Cities of the Americas: An Illustrated Encyclopedia, Santa Barbara, California, ABC-Clio, vol. 2, 2005, págs. 309-310.

38 AGS, SMA, leg. 0375, s.f.

39 Andrés Chereguini fue Ministro de la Marina entre 1732 y 1755. Sobre Chereguini véase el tomo XXXV, doc. 263 del Catálogo de la colección de documentos de Vargas y Ponce que posee el Museo Naval, serie primera, vol. II, Madrid, Instituto Histórico de Marina, 1979, pág. 27.

40 AGS, SMA, leg. 0375, s.f.

41 AGS, SMA, leg. 0375, s.f.

42 AGS, SMA, leg. 0375, s.f. 
por parte de las autoridades de poner nuevamente en práctica las obras para la reparación de las fuentes y cañería según el proyecto redactado por Maffey. Asimismo, es necesario apuntar que aunque los documentos consultados recogen con detalle y precisión los aspectos contemplados por Maffey para la ejecución de las obras que se debían emprender en el acueducto de Cartagena, no se hace referencia, ni se detalla con exactitud, la obra que finalmente se llevaría a cabo.

Junto al proyecto original, además, la documentación de 1732 da noticia de ocho capítulos adicionales presentados por el maestro Juan Baptista Guedea, redactados con el fin de mejorar las obras. No obstante, la falta de información sobre las modificaciones a la que solamente aluden estos ocho capítulos, y la ausencia de nuevos posibles documentos gráficos realizados durante la segunda fase del trabajo, impide profundizar y obtener conclusiones sobre el avance de las obras ejecutadas. Aún así, en 1732 el gobernador de Cartagena, don Juan Antonio de Ornedal y Maza, informaba a Patiño que se habían abastecido ocho fuentes de la ciudad con agua corriente, labores empezadas en $1730^{43}$. A pesar de esta noticia, tampoco es posible extrapolar el método empleado y la lógica constructiva que llevaría a introducir posibles modificaciones que pudieran hacer decantar la decisión de añadir estos nuevos ocho capítulos.

A todo esto hay que añadir que ignoramos si, en efecto, hubo una colaboración profesional entre Maffey y Feringán en la ejecución, por ejemplo, de los planos para la remodelación de las infraestructuras de Cartagena antes de 1724, cuando Maffey dejó la ciudad para ser designado a la costa atlántica. Feringán, en la nota adjunta a la copia del proyecto, no parece aludir a ningún cambio en la propuesta que realizó Maffey, hecho que demostraría respecto profesional y admiración hacia un estudio que refleja una minuciosidad loable. Maffey posee un buen conocimiento del terreno que procede de los años que había sido destinado a Cartagena, periodo en el que tuvo una participación activa en la elaboración del informe que ejecutó con gran celo y solvencia profesional y donde, además, el ingeniero italiano demostró unos esfuerzos para optimizar las soluciones propuestas. Su informe se basa en un programa funcional adecuado, optimiza los costes sin renunciar a la funcionalidad de la obra, apuntando a la necesidad de construir canales con material de alta calidad. El proceso culminaría algunos años después, pero aún así, y a la luz de los datos expuestos, la documentación tampoco deja reflejada de forma precisa la actuación de los ingenieros que intervinieron en la segunda fase de la restauración del acueducto de Cartagena, intervenciones que parecen realizarse y que podrían aflorar y ser respaldadas con argumentos más solidos en futuros trabajos de investigación.

43 AGS, SMA, leg. 0375, s.f. 
No obstante, se ha pretendido contextualizar apropiadamente el proyecto apoyándonos en la documentación gráfica existente, profundizando en los detalles y siguiendo las líneas de los criterios propuestos por Maffey que quiso, paulatinamente, adaptar a la realidad física del entorno, incidiendo en la restauración y/o reintegración, si lo fuese necesario, de las obras por realizar.

Este proyecto y, sobre todo, la construcción del arsenal cartagenero que implicó previamente la transformación del borde marítimo natural con el fin de crear la dársena del puerto al noroeste de la bahía ${ }^{44}$, obras que seguirán el proyecto de Maffey, reflejan la grandeza de la monarquía borbónica que patrocinó estas obras de ingeniería marítima. La solución constructiva propuesta por Maffey es acorde al momento histórico en el que se produce ${ }^{45}$. Representa un desafío técnico y científico, refleja su maestría y dominio del dibujo técnico en el proceso constructivo, es un reto técnico que se realiza dentro de un contexto histórico-arquitectónico que acomuna su propuesta con los trabajos de los restantes pragmáticos ingenieros militares responsables de las obras de remodelación del puerto de la ciudad según las exigencias de la monarquía.

44 Peñalver Martínez, La génesis.

45 Sobre la arquitectura y el urbanismo de la España «ilustrada» véase Carlos SAmbricıo, Territorio y ciudad en la España de la Ilustración, Madrid, Ministerio de Obras Públicas y Transportes, Centro de Publicaciones, 2 vols., 1991. 